\title{
Tratamiento de la Incontinencia Urinaria de Esfuerzo por la Operación de Burch
}

\author{
Doctores: Gabriel Alberto Tobón L., Gabriel Fernando Uribe E. y Jaime García M.
}

\section{INTRODUCCION}

La incontinencia urinaria es un grave problema en muchas mujeres que consultan en nuestros hospitales.

Es cada vez más frecuente que los ginecólogos se interesen en este problema, y "la línea divisoria anatómica entre la Ginecología y la Urología se ha derrumbado, por el interés común en la investigación y la asistencia de la incontinencia para beneficio de las dos disciplinas".

Son muchas las técnicas quirúrgicas que se han practicado y se siguen practicando en nuestro hospital para el tratamiento de la I.U.E. Desde 1977 se viene practicando la cirugía de Burch en nuestro hospital y presentamos los resultados.

Los aspectos que busca la cirugía son:

1. Restablecer la posición de la porción proximal de la uretra y permitir que se transmita la presión intraabdominal.

2. Aumentar la presión de cierre uretral.

3. Aumentar la longitud uretral funcional.

4. Restablecer la suficiencia del cuello vesical.

4. Aumentar el sostén para el cuello de la vejiga.

\section{OBJETIVO}

1. Analizar las cirugias por el método de Burch para la I.U.E. desde Octubre de 1977 a Junio de 1979.

2. Determinar edad y paridad de la paciente.

3. Determinar la evolución del tratamiento y sus complicaciones.

\section{I!I. UNIVERSO Y MUESTRA}

El universo y la muestra están constituidas por 15 pacientes que fueron atendidas en el servicio de Gineco-Obstetricia de Octubre de 1977 a Junio de 1979.

\section{PACIENTES Y METODO}

El material revisado consiste en 15 pacientes operadas en nuestro servicio; se hizo revisión de las historias, su evolución después del tratamiento y se $\mathrm{co}^{-}$ dificaron los resultados.

La técnica de la operación se ha normatizado y la describiremos a continuación:

1. Se coloca la paciente en posición horizontal de litotomía.

2. Se hace la asepsia del abdomen y de la vagina; se pasa una sonda de Folery N: 14 que va a servir durante la operación para delimitar el cuello vesical y la uretra; se introducen por la sonda de 10 a $15 \mathrm{ml}$ de agua. 
3. Se utiliza la incisión de Pfannenstiel o una mediana infraumbilical baja, se abre por planos piel, tejido celular subcutáneo y aponeurosis; se coloca separador abdominal.

4. Se hace disección roma del espacio de Retzius, se diseca bien la vejiga hasta llegar a la fascia paravaginal.

5. Luego el ayudante eleva los fondos de saco laterales por vagina, el cirujano los toma con pinzas de Allis y pasa dos puntos de Dexon $\mathrm{N}: 1$ a ambos lados, y los lleva luego al ligamento de Cooper donde se anudan, elevando el ayudante los fondos de saco para facilitar la elevación de la fascia paravaginal al anudar los puntos.

6. Posteriormente se hace la plicatura del ángulo-uretro-vesical.

7. Se hace una buena hemostasia y se procede al cierre de la pared; algunos cirujanos dejan dren. Se deja la sonda de Foley a permanencia de 5 a 8 días.

Usamos en las pacientes un antiséptico urinario mientras duren con la sonda.

\section{RESULTADOS}

Se obtuvieron los siguientes resultados:

EDAD, ESTADO CIVIL, PARIDAD.

El $86.7 \%$ fueron mayores de 40 años. el $13.3 \%$ tenían un promedio aritmético de 37.5 años. Iguales porcentajes se obtuvieron para el estado civil, casadas y solteras respectivamente.

Respecto a la paridad se encontró que el $60 \%$ tenian 7 o más hijos; el $40 \%$ restante un promedio de 3 hijos.

\section{Motivo de consulta y antecedentes}

El $93.3 \%$ de pacientes consultaron por su problema de incontinencia urinaria; el resto por prolapso. Un $46.7 \%$ tenía entre los antecedentes cirugias previas en el aparato genital, como histerectomías abdominales, operación de Manchester y plastias con puntos de Kelly.

También un sintoma predominante era la obesidad que se encontró en un 86.7 por ciento de las pacientes.

A todas las pacientes se les practicó cistouretrografía con cadena, el $93.3 \%$ tenía una incontinencia grado II en la clasificación de Green.

\section{CUADRO N: 1}

\section{TRATAMIENTO Y EVOLUCION}

Se ve en este cuadro que 9 pacientes mejoraron después del tratamiento; a 3 pacientes además del Burch, se les hizo la cirugia de Moscovich; 3 pacientes empeoraron después de la cirugía; una paciente mejoró pero posteriormente volvió a presentar la incontinencia y dos pacientes no se pueden evaluar por ser cirugías muy recientes.

(Cuadro No 1) TRATAMIENTO Y EVOLUCION

\begin{tabular}{|l|c|c|c|c|c|}
\hline \multicolumn{1}{|c|}{ Tratamiento Quirúrgico } & \multicolumn{5}{|c|}{ Evolución } \\
\hline & mejoró & empeoró & $(3)$ & $(4)$ & total \\
\cline { 2 - 6 } & 6 & 3 & 1 & 2 & 12 \\
Operación de Burch & 3 & - & - & - & 3 \\
Operación de Moscovich & 9 & 3 & 1 & 2 & 15 \\
\hline TOTAL & & \multicolumn{3}{|c|}{} \\
\hline
\end{tabular}

(3) mejoró y posteriormente...

(4) no se pueden evaluar por ser de reciente ocurrencia. 


\section{CUADRO N: 2 \\ COMPLICACIONES}

Las complicaciones que se han tenido son sintomatología de infección urinaria en 5 pacientes, sólo una con urocultivo positivo, pero creemos que estas infecciones son debidas a la sonda a permanencia; mejoraron con tratamiento médico .

En dos pacientes hubo ruptura de vejiga al pasar los puntos, creemos que es debido a una disección ro completa de la vejiga.

(Cuadro No.2)

\begin{tabular}{|lr|}
\hline \multicolumn{2}{|c|}{ COMPLICACIONES } \\
\hline Infecciones urinarias & 5 \\
Ruptura de vejiga & 2 \\
Eventración & 1 \\
Acadamiento de uréter en riñón & 1 \\
No hubo & 6 \\
\hline TOTAL & 15 \\
\hline
\end{tabular}

En una paciente se presentó eventración, en otra hubo un acodamiento del uréter que se comprobó por urografía excretora presentando exclusión del riñón, se volvió a intervenir soltando el punto de ese lado y en una urografía de control posterior a la cirugía, se visualizó nuevamente el riñón funcionando bien.

\section{COMENTARIOS}

Es de pensar que mostrando este pequeño trabajo interesamos a los colegas ginecólogos a prestar más atención al tratamiento de la Incontinencia Urinaria de Esfuerzo.

Una vez lograda la práctica en la operación el tiempo aproximado es de una hora.
El diagnóstico y tratamiento de la Incontinencia Urinaria de Esfuerzo, han sido extensamente analizados por los diversos autores, estamos conscientes de que un extenso estudio cronológico será lo ideal para llegar a un correcto diagnóstico; pero las limitaciones de nuestro medio nos obligan a utilizar la historia clínica, urocultivo y la cistouretrografía con cadena, como exámenes fundamentales; pensamos que las cistoscopia y cistometría serían exámenes de mucha utilidad para el estudio de esas pacientes, la urografía excretora y las diversas técnicas de uretroscopia sólo serían útiles en casos especiales.

Con respecto al tratamiento creemos que una vez hecho el diagnóstico correcto de Incontinencia Urinaria de Esfuerzo anatómica verdadera, la técnica de elección es la uretropexia retropúbica por la técnica de pared vaginal. Como es una técnica relativamente nueva es un común denominador en todos los servicios que manejan estos problemas y en el nuestro, ir modificando paulatinamente la técnica hasta encontrar el procedimiento ideal que brinde el mayor porcentaje de curaciones y el menor número de complicaciones.

No estamos satisfechos plenamente con los resultádos y con el número de complicaciones, lo cual indica que aún necesitamos más experiencia en el manejo de esta entidad.

Pensamos que el mismo cirujano puede hacer todo el procedimiento. Tal vez un punto a cada lado sea suficiente (últimamente nos quedan dudas sobre el tipo de material a usar) y, por tanto, creemos que el mayor éxito y la clave para evitar las complicaciones consiste en la adecuada disección para visualizar la fascia paravaginal.

Siendo este un trabajo preliminar nos proponemos hacer un protocolo para una técnica única con el fin de analizar uniformemente la paciente. 


\section{RESUMEN}

Se analizan 15 pacientes con incontinencia urinaria de esfuerzo vistas en el Servicio de Ginecología del Hospital universitario San Vicente de Paúl, a quienes se les practicó la técnica Burch; se describe esta, se discuten sus ventajas e inconvenientes

\section{TREATMENT OF URINARY EFFORT INCONTINENCE WITH BURCH'S OPERATION}

\section{SUMMARY}

An analysis was made on 15 patients with Urinary Effort Incontinence, who were examined at the Ginecology Service of Hospital Universitario San Vicente de Paul and treated with Burch's technique Such technique is described and its advantages and inconveniencies discussed.

\section{Bibliografía}

1. BURCH J. C.: Urethrovesical fixtation to Cooper's ligament for correction of strees incontinence, cystocele and prolapse. Am J. Obstet Gynecol 81: 281, 1961.

2. BURCH J. C.: Cooper's ligament uretrovesical suspension for strees incontinence. Nine years experience-results, complication, technique, Am J. Obstet Gynecol 100: 764, 1968.

3. GREEN, T. H. Development of a plan the diagnosis and treatment of urinary strees incontinence. American Journal of Obstet and Gynecol, 83: 632-648, 1962.

4. GREEN, T. H. Urinary stress incontinence: Differential diagnosis, pathophysiology and manegement American Journal of Obstet and Gynecol 112: 368-400, 1975.

5. DRUKKER B., MILLER D. Uretropexia retro. púbica por la técnica de pared vaginal en la incontinencia urinaria de alarma. Clínicas Obstétricas y Ginecológicas 3: 813-823, 1978.

6. STANTON, S. Cirugía en la incontinencia urinaria. Ginecología y Obstetricia Interamericana 79-103, 1978. 